(NASA-CR-199324) THE MINIMAL TIME DETECTION ALGORITHM (California N96-15989 Univ.) $13 \mathrm{p}$

Unclas

$63 / 61 \quad 0065148$ 


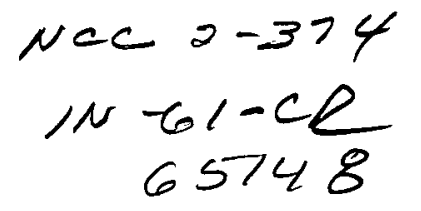

\title{
The Minimal Time Detection Algorithm P $_{-} 13$
}

\author{
Sungwan Kim \\ Electrical Engineering Department \\ University of California, Los Angeles
}

Abstract - An aerospace vehicle may operate throughout a wide range of flight environmental conditions that affect its dynamic characteristics. Even when the control design incorporates a degree of robustness, system parameters may drift enough to cause its performance to degrade below an acceptable level. The object of this paper is to develop a change detection algorithm so that we can build a highly adaptive control system applicable to aircraft systems. The idea is to detect system changes with minimal time delay. The algorithm developed is called Minimal Time-Change Detection Algorithm(MT-CDA) which detects the instant of change as quickly as possible with false-alarm probability below a certain specified level. Simulation results for the aircraft lateral motion with a known or unknown change in control gain matrices, in the presence of doublet input, indicate that the algorithm works fairly well as theory indicates though there is a difficulty in deciding the exact amount of change in some situations. One of MT-CDA distinguishing properties is that detection delay of MT-CDA is superior to that of Whiteness Test.

\section{Introduction}

We wish to design an aerospace vehicle control system which operates throughout a wide range of flight environmental conditions that affect its dynamic characteristics. The problem of identifying aircraft system parameters from flight test data and their application to flight control has been successfully solved for linear models, by Balakrishnan [2], Iliff [11], and Maine [15], [16], when there are no severe changes in aircraft and environment. Even when the control design incorporates a degree of robustness, system parameters may drift enough to degrade its performance below an acceptable level. In other words, we still need to develop a theory for a better control system when aircraft flies at a high angle of attack where the linear model is no longer valid and when the robust controller does not work due to large variations in parameters or noise levels.

Adaptive controls are considered to be a promising approach to give us a possible solution. An adaptive control problem arises whenever system parameters are unknown or are subject to unknown variation which is usually a small (or smooth) change. It is also of increasing practical importance, since adaptive techniques are being used more and more in industrial control systems. However, this field is not mature yet, and only a set of scattered simulational results exists with no single quantitative measure for judging performances [1]. Taking into account abrupt (or drastic) changes in statistical models appears as a natural complement of most of the adaptive techniques which track only slow variations of parameters. So, the detection of abrupt change is essential in the design of

\footnotetext{
${ }^{1}$ This work was supported by NASA Ames Research Center, DFRF through UCLA Flight Systems Research Center(FSRC), Grant Number NCC 2-374.
} 
an adaptive system for these types of adaptive control problems. Therefore, the problem of detection, estimation, and diagnosis of changes in dynamical properties of signals or systems are considered, with particular emphasis on statistical methods for detection, to provide a general framework for change detection in signals and systems.

The development of the change detection problem was stimulated by Wald [25] in 1947 when 'Sequential Analysis' was published and a Sequential Probability Ratio Test(SPRT) was introduced. In 1954, a 'sequential process inspection scheme' was proposed by Page [18], [19] to detect a change in the mean by testing a weighted sum of the last few observations, i.e. a moving average. Named the 'Page-Hinkley' rule, Page pointed out that this rule is equivalent to performing a SPRT. The theoretical properties of this rule have been investigated for a long time from on-line and off-line points of view. The most significant works in that direction are Shiryayev [21] and Lorden [14]. They formulated an 'Optimal Stopping (or Detection)' problem and proposed simple rules which are optimal (or asymptotically optimal) in an appropriate sense. And the general problem of optimality of random processes in discrete time was considered and developed by Chow, Robbins, and Siegmund [7]. The problem of change detection has received growing attention during the last 20 years, as can be seen from the survey papers (Wilsky [27] and Basseville [4]), the books (Basseville and Benvensite [5], Basseville and Nikiforov [6]) and so on. Further discussions may be found in these References.

The development of change detection method is still a relatively new subject. Presently, most of the work has been at a theoretical level with only a few real applications of techniques: edge detection in pattern recognition, segmentation of speech (or image) signal, biomedical signal monitoring, vibration monitoring, quality control, fault-detection, etc.

As mentioned earlier, an algorithm is required to adapt to new circumstances for a total adaptive system applicable to the aircraft control systems. One important new idea is an algorithm to detect system change with minimal time delay that involves fairly advanced stochastic theory. This could be formulated based on the problem of an optimal stopping rule [21] in which we seek to detect the instant of change as quickly as possible, subject to the given false-alarm probability.

A block diagram shown in Figure 1 explains the total adaptive system for the aircraft control that is equipped with a CDA. Before the CDA detects a change, the regulator is designed based on the initial parameter values. But after the change is detected at time $\tau$, the CDA block generates an Activation Signal to re-initiate system identification. The system identification process finds a new set of parameter values proper for the changed system. The regulator uses the new parameter values to build a new control law. This process is continued until the new parameter set converges. So, a total adaptive control system can be designed that is good for a system that undergoes changes.

Algorithm complexity is definitely an issue for detection of each possible change and time of change. In spite of this complexity, we can have a control system which can detect its change and adapt it as quickly as possible even though the large amount of change is occurred at random time. Therefore, this MT-CDA will present a proper starting point for total adaptive control system in the theoretical point of view. 


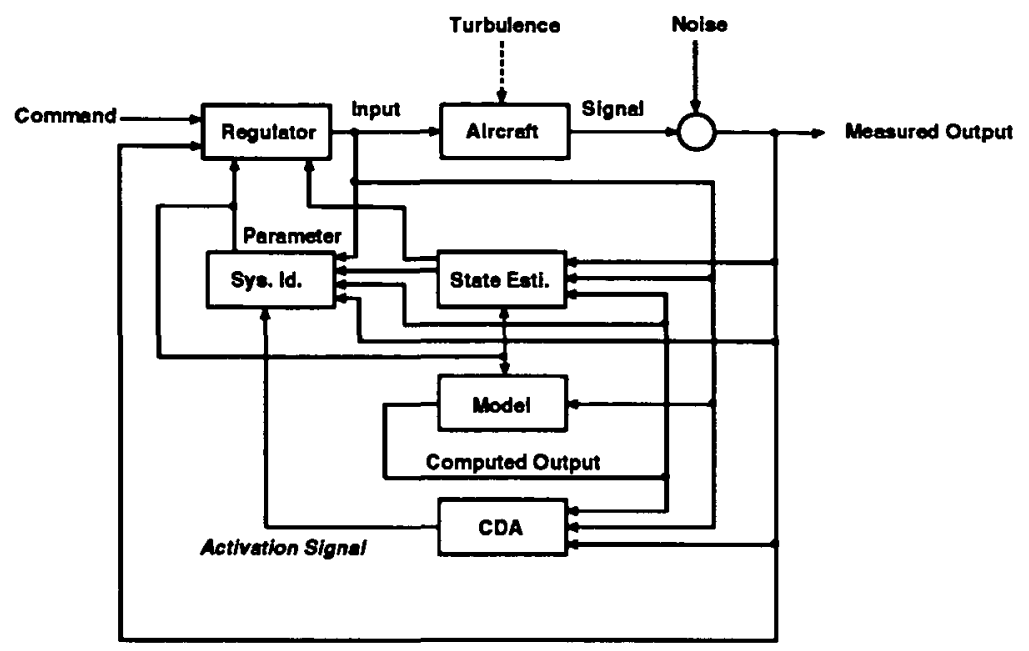

Figure 1: A Block Diagram of Control System for Aircrafts with CDA

2. Minimal Time-Change Detection Algorithm

\subsection{MT-CDA: Detection of a Known Change}

The following state space model is considered.

$$
\begin{gathered}
x_{n+1}=A x_{n}+\left(B_{1}+\left(B_{2}-B_{1}\right) \chi(n-\theta)\right) u_{n} \\
v_{n}=C x_{n}+\left(D_{1}+\left(D_{2}-D_{1}\right) \chi(n-\theta)\right) u_{n}+G N_{n} \\
\chi(m)=\left\{\begin{array}{lll}
0 & \text { if } & m<0 \\
1 & \text { if } & m \geq 0
\end{array}\right.
\end{gathered}
$$

Based on the Eq.(1) and (2), the basic problem to be solved is to detect a known change in control gain matrices $(B$ and $D)$ as quickly as possible in order to build an highly adaptive system whose parameters $(B$ and $D)$ could be switched from a known system to another known system after change detection. This is appropriate for a system which operates in two alternate driving points, and restricted to the class of models with a change of parameters which is known. The probability of making a false (or wrong) detection also needs minimized since the signals are disturbed by a white Gaussian noise.

We want to formulate a problem which is to find the detection time $(\tau)$ to minimize the average delay

$$
E\left[(\tau-\theta)^{+}\right]
$$

subject to a given false alarm probability, i.e.

$$
\operatorname{Pr} .[\tau<\theta] \leq \alpha
$$


where $\alpha$ is given. 'Likelihood Ratio (change vs. no change)', which we shall define to be the ratio

$$
\frac{\text { Joint density of } v_{1}, \cdots, v_{n} \text { (with change) }}{\text { Joint density of } v_{1}, \cdots, v_{n} \text { (without change) }}
$$

is introduced and $p(\cdot), p_{\infty}(\cdot)$ denote the likelihood probability density function with and without changes at $\theta=k$.

$$
\begin{gathered}
p\left(v_{1}, v_{2}, \cdots, v_{n} \mid \theta=k\right)=p\left(v_{1}, \cdots, v_{k}, v_{k+1}, \cdots, v_{n} \mid \theta=k\right) \\
p_{\infty}\left(v_{1}, v_{2}, \cdots, v_{n}\right)=p\left(v_{1}, \cdots, v_{n} \mid \theta>n\right)
\end{gathered}
$$

Then we define the following functional

$$
L_{n, k}=2 \log \frac{p\left(v_{1}, v_{2}, \cdots, v_{n} \mid \theta=k\right)}{p_{\infty}\left(v_{1}, v_{2}, \cdots, v_{n}\right)} .
$$

and for each $n$,

$$
\ell_{n}=\max _{k \leq n} L_{n, k}
$$

Now a decision rule for CDA is

$$
\tau=\min \left\{n: \ell_{n}>h\right\}
$$

where $\tau$ is a change detection time and $h$ is a threshold value computed from the probability of false alarm equation(Eq.(5)).

This algorithm stops the first time when $\ell_{n}$ exceeds a given threshold. This is based on Eq.(8)-(10), so it can be regarded as 'Maximum Likelihood' treatment of the unknown change time, i.e. stop at $\tau=n$ when for some $k$ the observations $v_{k}, \cdots, v_{n}$ are significant.

By the contributions of earlier works of Shiryayev [21], Lorden [14], Bansal [3], and Tartakovsky [23], [24] this procedure could be said optimal (at least asymptotically optimal), in the sense that it minimizes the detection delay for a fixed false alarm probability.

\subsection{MT-CDA: Detection of an Unknown Change}

In this section, minimal time detection of an unknown change instead of a known change is considered, and its algorithm is derived. The detection of an unknown change is a more complicated problem than the detection of a known change because there is no information on the amount of change. Multiple detectors are required to detect an unknown change, where the each detector is based on the known amount of change, in contrast to single detector required for the detection algorithm of a known change. In Section 2.2.1, the algorithm for the detection of only change time, which is a little bit simpler problem, is first derived and the detection algorithm of both time and amount of change is followed in Section 2.2.2.

2.2.1. Detection of the Time (or Instant) of Change: The detection of change time is a significant problem in some applications, for example, 
1. Fault detection : all the processes are stopped when a failure occurs and are restarted after the failure is fixed.

2. When a system requires the exact parameter set after a change, it is preferable to re-initiate the system identification just after detecting a change.

In adaptive system sense, the 2 nd case is a popular one and a promising problem to be considered [9]. To detect an unknown change, the CDA Block in Figure 1 contains multiple change detectors. Each of (CDA $)_{i}$ is based on a fixed known change, so each of detectors is basically the same one as derived in Section 2.1. By examining the Activation Signal for each $n$, the real Activation Signal at time $n$ is generated by the earliest one. The idea of this algorithm is that if there exists an unknown change, the exact (or the closest) detector will make Activation Signal at the earliest time. So the time of an unknown change will be detected with this algorithm if the amount of an unknown change is in the range of the multiple detector hypotheses.

To formulate this MT-CDA for an unknown change, we consider the following system model

$$
\begin{gathered}
x_{n+1}=A x_{n}+\left(B_{1}+\left(B_{2}-B_{1}\right) \chi(n-\theta)\right) u_{n} \\
v_{n}=C x_{n}+\left(D_{1}+\left(D_{2}-D_{1}\right) \chi(n-\theta)\right) u_{n}+G N_{n} \\
B_{2}, D_{2} \cdots \text { unknown. }
\end{gathered}
$$

The only difference, compared to the Section 2.1 , is that control gain matrices $\left(B_{2}\right.$ and $D_{2}$ ) are unknown which stands for the unknown change. Like Section 2.1, we define the following functional, which is related to 'Likelihood Ratio', for each detector

$$
L_{n, k}^{i}=2 \log \frac{p\left(v_{1}, v_{2}, \cdots, v_{n} \mid \theta=k, B_{2}=B_{2}^{i}, D_{2}=D_{2}^{i}\right)}{p\left(v_{1}, v_{2}, \cdots, v_{n} \mid \theta>n\right)} .
$$

where each superscript ' $i$ ' stands for $i$-th detector $(i=1,2, \cdots, N)$ and for each $n$ and each detector,

$$
\ell_{n}^{i}=\max _{k \leq n} L_{n, k}^{i} .
$$

Then the decision rule for the detection of an unknown change is

$$
\begin{gathered}
\tau^{i}=\min \left\{n: \ell_{n}^{i}>h^{i}\right\} \\
\tau=\min _{i} \tau^{i}
\end{gathered}
$$

where $\tau^{i}$ is a change detection time of each detector, $\tau$ is a real change detection time, and $h^{i}$ is a threshold value for each detector which is found from the probability of false 
alarm equation. So we can detect this kind of unknown change at time $\tau$ which is the earliest detection time among all the multiple detectors considered in this algorithm. To have a good detection algorithm, the number of change detectors $(N)$ should be sufficiently large and the dynamic range of detectors' change amount should be sufficient to cover the estimated range of changes in system parameters. This means that there exists a tradeoff relationship between 'Performance' and 'Complexity' of the algorithm because the computational burden increases dramatically as the number of change detectors increases.

2.2.2. Detection of the Time and Amount of Change: In some applications, it is insufficient to detect only the change time if the change is unknown, it is also required to detect the amount of change. Figure 2 is a modified block diagram of Figure 1 for this purpose. $\overline{\text { CDA }}$ Block is a change detection algorithm which can estimate the amount of an unknown change and the change time. It generates the $\operatorname{Activation} \operatorname{Signal}(A S)$ and an Estimated Degree of Change $(E D C)$ which is closely related to the real amount of change limited by the resolution of the detectors. When the system change is detected, the system parameter set is updated based on EDC and Parameter Update Block that rules the parameter update law. There is no necessity of System Identification routine. This looks simpler than the adaptive control system explained in Section 2.2.1, but the $\overline{\mathrm{CDA}}$ Block is much more complicated than CDA Block. So, Figure 2 could be considered another version of block diagram for the total adaptive control system that is appropriate for a system that undergoes an unknown change in parameters. To determine the amount

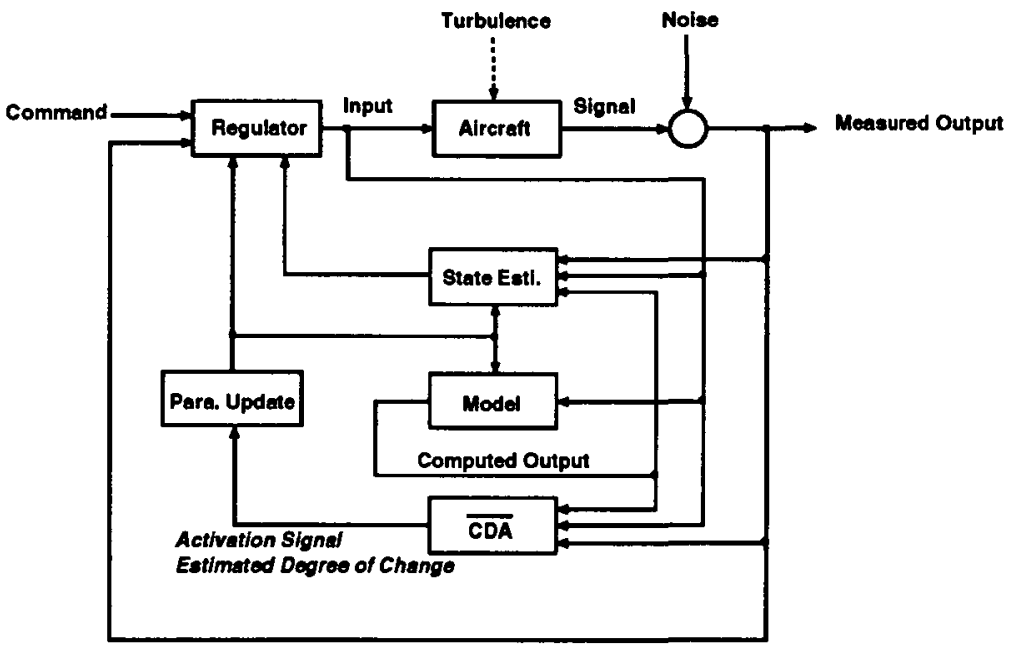

Figure 2: A Block Diagram of Control System for Aircrafts with $\overline{\mathrm{CDA}}$

of change, a 'Decision Mechanism' is required. Based on the $A S$, each of that comes from a known change detector, the Decision Mechanism generates a real one and an $E D C$ at time $n$. The algorithm formulation is the exactly same as Section 2.2.1 (Eq.(11)-(16)) except the Decision Mechanism, so only the details of Decision Mechanism is explained in the following.

$$
\tau^{i}=\min \left\{n: \ell_{n}^{i}>h^{i}\right\}
$$




$$
\begin{gathered}
\tau=\min _{i} \tau^{i} \\
\kappa=\min \left\{i: \tau^{i}\right\} .
\end{gathered}
$$

We can then detect an unknown change at time $\tau$ whose $E D C$ is equivalent to the $\kappa$-th detector, if the amount of an unknown change is in the range of the amount of multiple detectors. The difficulty of the decision of the exact EDC exists in decision rule(Eq.(17)(19)), called 'D.R. \# 1' hereafter, because some of change detectors could respond at the same time. That means the necessity of a better decision rule. Because the exact value of $\ell_{n}^{i}$ ('exact' means the exact or the closest change detector corresponding to the given unknown change) is rapidly increasing, the following new decision rule, called 'D.R. \# 2', could be proposed.

$$
\begin{gathered}
\tau^{i}=\min \left\{n: \ell_{n}^{i}>h^{i}\right\} \\
\tau=\min _{i} \tau^{i} \\
\kappa= \begin{cases}\max \left\{i:\left(\ell_{n}^{i}-h^{i}\right)\right\} & \text { if } \exists \text { more than } 2 \text { minimum } \tau^{i} \\
\min \left\{i: \tau^{i}\right\} & \text { otherwise. }\end{cases}
\end{gathered}
$$

There exists the same trade-off between performance and the complexity of the algorithm explained in Section 2.2.1. Only a finite number of change detectors are used in real simulations.

\section{Simulations and Results}

A lateral rigid body dynamics of PA-30, which is a Linear Time-Invariant(LTI), is used for simulations [12], [17].

\subsection{Detection of a Known Change}

The mean $(M[\cdot])$ and the variance $(V[\cdot])$ of detection delay $(\tau-\theta)$, with respect to the different change time $(\theta)$, based on 100 runs hereafter, are summarized in Table $1.50 \%$ change is detected earlier than $25 \%$ or $10 \%$ change. From the result of Table 1 , it is shown that different known changes could been detected with some delays.

\subsection{Detection of an Unknown Change: Time only}

Simulations with 6 change detectors are done first and then combined to analyze their results as summarized in Table 2 . From this Table, the followings are observed:

- For a given detector, large amount of change is detected earlier than small amount of change. 


\begin{tabular}{||c||c|c|c|c|c|c|c|c|c|c||}
\hline \hline$\theta$ & 10 & 20 & 30 & 40 & 50 & 60 & 70 & 80 & 90 & 100 \\
\hline$M[\tau-\theta]$ & 7.6 & 0.9 & 7.1 & 4.0 & 11.7 & 10.3 & 11.4 & 12.2 & 11.7 & 12.1 \\
\hline$V[\tau-\theta]$ & 0.4 & 0.3 & 4.5 & 0.4 & 26.6 & 8.2 & 11.7 & 12.2 & 13.3 & 18.2 \\
\hline \hline
\end{tabular}

(a) $10 \%$ Change

\begin{tabular}{||c||c|c|c|c|c|c|c|c|c|c||}
\hline \hline$\theta$ & 10 & 20 & 30 & 40 & 50 & 60 & 70 & 80 & 90 & 100 \\
\hline$M[\tau-\theta]$ & 6.2 & 0.1 & 3.1 & 3.0 & 1.6 & 4.6 & 4.8 & 5.2 & 5.1 & 5.0 \\
\hline$V[\tau-\theta]$ & 0.5 & 0.1 & 0.6 & 0.1 & 1.9 & 1.0 & 1.6 & 1.4 & 1.8 & 1.7 \\
\hline \hline
\end{tabular}

(b) $25 \%$ Change

\begin{tabular}{||c||c|c|c|c|c|c|c|c|c|c||}
\hline \hline$\theta$ & 10 & 20 & 30 & 40 & 50 & 60 & 70 & 80 & 90 & 100 \\
\hline$M[\tau-\theta]$ & 4.5 & 0.0 & 1.4 & 2.2 & 0.2 & 2.3 & 2.5 & 2.8 & 2.7 & 2.7 \\
\hline$V[\tau-\theta]$ & 0.7 & 0.0 & 0.3 & 0.2 & 0.1 & 0.5 & 0.6 & 0.5 & 0.5 & 0.5 \\
\hline \hline
\end{tabular}

(c) $50 \%$ Change

Table 1: Simulation Results for Various Change Times when Change is in Rolling Motion

- Rolling motion :

$-10 \%$ Change: $50 \% \mathrm{D} \geq 25 \% \mathrm{D} \geq 10 \% \mathrm{D}$

- 25\% Change: $10 \% \mathrm{D}, 50 \% \mathrm{D} \geq 25 \% \mathrm{D} \Rightarrow 10 \% \mathrm{D}, 50 \% \mathrm{D} \simeq 25 \% \mathrm{D}$

$-50 \%$ Change: $10 \% \mathrm{D} \geq 25 \% \mathrm{D} \geq 50 \% \mathrm{D} \Rightarrow 10 \% \mathrm{D} \simeq 25 \% \mathrm{D} \simeq 50 \% \mathrm{D}$

where ' $\mathrm{D}$ ' denotes a detector and the detection delay is quite reduced when $10 \%$ detector is used to detect $10 \%$ change but there is no big difference in detection delay of 3 change detectors to detect $25 \%$ or $50 \%$ change.

- Yawing motion : The results are the same as rolling motion, but detection delay is large and the difference of delay with respect to each change detector is small since the portion of yawing motion in PA-30 is small.

From the above observations and the trade-off between the number of change detectors and the computational burden, it could be concluded that $10 \%$ change detector is sufficient when the detection of change larger than $10 \%$ is concerned.

\subsection{Detection of an Unknown Change: Time and Amount}

Simulation results, with D.R. \# 2, for $\theta=40$ are summarized in Table 3. From this table, it could be shown that the unknown changes at $\theta=40$ are fairly well detected except $30 \%$ 


\begin{tabular}{||l||c|c|c|c|c|c||}
\hline \hline \multicolumn{1}{||c||}{} & \multicolumn{2}{c|}{$10 \%$} & \multicolumn{2}{c||}{$25 \%$} & \multicolumn{2}{c||}{$50 \%$} \\
\cline { 2 - 7 } & R.D. & Y.D. & R.D. & Y.D. & R.D. & Y.D. \\
\hline \hline $10 \%$ R.C. & 7.05 & 16.16 & 9.02 & 15.84 & 12.31 & 15.71 \\
\hline $10 \%$ Y.C. & N.D. & 16.61 & N.D. & 16.61 & N.D. & 16.49 \\
\hline $20 \%$ R.C. & 3.88 & 15.61 & 3.84 & 15.58 & 4.31 & 15.0 \\
\hline 20\% Y.C. & N.D. & 16.61 & N.D. & 16.60 & N.D. & 16.48 \\
\hline $25 \%$ R.C. & 3.23 & 15.17 & 3.11 & 15.01 & 3.29 & 14.91 \\
\hline 25\% Y.C. & N.D. & 16.61 & N.D. & 16.60 & N.D. & 16.48 \\
\hline $30 \%$ R.C. & 2.73 & 15.02 & 2.55 & 14.88 & 2.73 & 15.85 \\
\hline 30\% Y.C. & N.D. & 16.61 & N.D. & 16.59 & N.D. & 16.47 \\
\hline $40 \%$ R.C. & 2.18 & 14.79 & 1.90 & 14.73 & 1.94 & 14.77 \\
\hline 40\% Y.C. & N.D. & 16.61 & N.D. & 16.56 & N.D. & 16.46 \\
\hline $50 \%$ R.C. & 1.81 & 14.66 & 1.57 & 14.68 & 1.43 & 14.70 \\
\hline 50\% Y.C. & N.D. & 16.61 & N.D. & 16.56 & N.D. & 16.45 \\
\hline $60 \%$ R.C. & 1.55 & 14.54 & 1.25 & 14.63 & 1.11 & 14.64 \\
\hline 60\% Y.C. & N.D. & 16.56 & N.D. & 16.51 & N.D. & 16.45 \\
\hline \hline
\end{tabular}

Table 2: Summarized Simulation Results for $\theta=30$
R.D. $\cdots$ Rolling Detector
Y.D. $\quad \cdots$ Yawing Detector
R.C. $\cdots$ Rolling Change
Y.C. $\cdots$ Yawing Change
N.D. $\cdots$ No Detection

and $40 \%$ changes, which might be fixed if the better decision rule is applied. Simulation results for $\theta=10,20$, and 30 have the same characteristics.

Therefore, it could be concluded that the given unknown changes are detected in statistical sense, though there exists a difficulty in deciding the exact $E D C$ in some situations which might be improved by applying the better decision rule.

\subsection{Change Detection via Whiteness Test}

To examine the validity of MT-CDA, derived in Section 2.1 and 2.2, simulations of Whiteness Test are done and results are compared in this section. Only the detection problem of a known change is considered here.

Table 4 shows the detection delay of each algorithm for various change time $(\theta)$. For every tests, the detection delay of MT-CDA is smaller than that of Whiteness Test. Therefore, the simulation results show that MT-CDA is superior to Whiteness Test in the sense of detection delay comparison. 


\begin{tabular}{||c||c|c|c||}
\hline \hline & Detection Number & $M[\tau-\theta]$ & $V[\tau-\theta]$ \\
\hline \hline 10\% D & 69 & 4.12 & 0.31 \\
\hline 20\% D & 29 & 3.55 & 0.39 \\
\hline 30\% D & 0 & & \\
\hline 40\% D & 1 & 4.0 & 0.0 \\
\hline 50\% D & 1 & 2.0 & 0.0 \\
\hline \hline
\end{tabular}

(a) $10 \%$ change

\begin{tabular}{||c||c|c|c||}
\hline \hline & Detection Number & $M[\tau-\theta]$ & $V[\tau-\theta]$ \\
\hline \hline $10 \%$ D & 0 & & \\
\hline 20\% D & 87 & 3.15 & 0.15 \\
\hline 30\% D & 3 & 3.33 & 0.22 \\
\hline 40\% D & 3 & 3.0 & 0.0 \\
\hline $50 \%$ D & 7 & 2.29 & 0.49 \\
\hline \hline
\end{tabular}

(b) $20 \%$ change

\begin{tabular}{||c||c|c|c||}
\hline \hline & Detection Number & $M[\tau-\bar{\theta}]$ & $V[\tau-\theta]$ \\
\hline \hline 10\% D & 0 & & \\
\hline 20\% D & 18 & 2.89 & 0.10 \\
\hline 30\% D & 27 & 3.07 & 0.07 \\
\hline 40\% D & 33 & 2.97 & 0.03 \\
\hline 50\% D & 22 & 2.23 & 0.18 \\
\hline \hline
\end{tabular}

(c) $30 \%$ change

\begin{tabular}{||c||c|c|c||}
\hline \hline & Detection Number & $M[\tau-\theta]$ & $V[\tau-\theta]$ \\
\hline \hline 10\% D & 0 & & \\
\hline 20\% D & 4 & 2.5 & 0.25 \\
\hline 30\% D & 3 & 3.0 & 0.0 \\
\hline 40\% D & 23 & 3.0 & 0.0 \\
\hline 50\% D & 70 & 2.36 & 0.23 \\
\hline \hline
\end{tabular}

(d) $40 \%$ change

\begin{tabular}{||c||c|c|c||}
\hline \hline & Detection Number & $M[\tau-\theta]$ & $V[\tau-\theta]$ \\
\hline \hline $10 \%$ D & 0 & & \\
\hline 20\% D & 1 & 2.0 & 0.0 \\
\hline $30 \%$ D & 0 & & \\
\hline 40\% D & 2 & 2.0 & 0.0 \\
\hline $50 \%$ D & 97 & 2.23 & 0.18 \\
\hline \hline
\end{tabular}

(e) $50 \%$ change

Table 3: Simulation Results for $\theta=40$ 


\begin{tabular}{||c||c|c|c|c|c|c|c|c|c||}
\hline \hline$\theta$ & 10 & 20 & 30 & 40 & 50 & 60 & 70 & 80 & 90 \\
\hline$M[\tau-\theta]$ & 6.2 & 0.1 & 3.1 & 3.0 & 1.6 & 4.6 & 4.8 & 5.2 & 5.1 \\
\hline$V[\tau-\theta]$ & 0.5 & 0.1 & 0.6 & 0.1 & 1.9 & 1.0 & 1.6 & 1.4 & 1.8 \\
\hline \hline
\end{tabular}

(a) Minimal Time-Change Detection Algorithm

\begin{tabular}{||c||c|c|c|c|c|c|c|c|c||}
\hline \hline$\theta$ & 10 & 20 & 30 & 40 & 50 & 60 & 70 & 80 & 90 \\
\hline$M[\tau-\theta]$ & 11.7 & 4.0 & 17.9 & 7.9 & 37.4 & 33.0 & 32.4 & 30.6 & 28.4 \\
\hline$V[\tau-\theta]$ & 0.2 & 0.0 & 0.1 & 0.1 & 4.8 & 3.7 & 4.4 & 5.9 & 5.4 \\
\hline \hline
\end{tabular}

(b) Whiteness Test

Table 4: Simulation Results for Various Change Times when 25\% Change is in Rolling Motion

\section{Conclusions}

A simple model with a single change in parameters of control gain matrices $(B$ and $D)$ at random time whose a priori distribution is known has been considered. Changes in these matrices mean the failure in the actuators, so possible damages in the control surfaces (aircraft wings) and/or one of the engine failure could be analyzed with this model. Therefore, considering changes in the control gain matrices is the proper starting point before we consider all kinds of changes. MT-CDA, based on Likelihood Ratio Test, for this simple model has been developed whether a change is known or unknown and it has been shown to be optimal for a fixed false alarm probability.

Simulation results for lateral motions show that a known change can be detected with some delays which should be minimal for the given condition as theory indicates. The detection problem of an unknown change is much more complicated because there is no information on the amount of change. It requires multiple change detectors where each detector is based on the known amount of change. This problem is considered in two ways: the detection of change time only and the detection of both time and amount of change. The former algorithm is a little bit simple but it requires a system identification routine to build a total adaptive system. Due to the trade-off between the performance and the complexity (number of change detectors), only a finite number of change detectors are used for simulations. From simulation results for the detection of change time, it could be concluded that $10 \%$ change detector, which is the smallest one, is sufficient and it is robust in the sense that it could detect any change $(>10 \%)$ with sub-minimal detection delay. And the unknown change is detected, in statistical sense, though there exists a difficulty to decide the exact $E D C$ in some situations. The better (or best) decision rule needs to be developed to solve this difficulty. The algorithm with multiple change detectors, if implemented in full, will yield the best performance for the widest class of change. But full implementation is another problem. Simulations have been done throughout the 
various situations and the results are meaningful. Detection via Whiteness Test has been also formulated for algorithm comparison in the sense of detection delay. MT-CDA is superior to the Whiteness Test in this sense.

In this paper, MT-CDA has been developed and demonstrated by simulations when a change is in control gain matrices. With this MT-CDA, a total adaptive control system shown in Figure 1 or Figure 2 could be built and work throughout a wide range of conditions.

\section{References}

[1] Åström, K.J. and Wittenmark, B.:Adaptive Control, Addison-Wesley Publishing Co., 1989

[2] Balakrishnan, A.V.: Identification and Adaptive Control: An application to Flight Control Systems,Journal of Optimization Theory and Applications, Mar., 1972

[3] Bansal R.K. and Papantoni-Kazakos P.: An Algorithm for Detecting a Change in a Stochastic Process, IEEE Transactions on Information Theory, Vol. IT-32, No. 2, pp. 227-235, Mar. 1986

[4] Basseville, M.: Detecting Changes in Signals and Systems - A Survey, Automatica, Vol. 24, No. 3, pp. 309-326, 1988

[5] Basseville, M. and Benveniste, A.: Detection of Abrupt Changes in Signals and Dynamical Systems, Lecture Notes in Control and Information Sciences 77, SpringerVerlag, 1980

[6] Basseville, M. and Nikiforov I.V.: Detection of Abrupt Changes:Theory and Application, Englewood Cliffs, Prentice Hall, New Jersey, 1993

[7] Chow, Y.S., Robbins H., and Siegmund, D.: Great Expectations:The Theory of Optimal Stopping, Houghton Mifflin, Boston, 1971.

[8] Davis, M.H.A.: The Application of Nonlinear Filtering to Fault Detection in Linear Systems, IEEE Transactions on Automatic Control, Vol. AC-20, No. 2, pp. 257-259, Apr. 1975

[9] R. Fernandez d. B. et al.: Parameter Transient Tracking for Recursive Estimation, IEE Proceedings-D, Vol. 138, No. 1, pp. 68-74, Jan. 1991

[10] Gibbs, B.P.: Jump Detection/Estimation Using Stepwise Regression, IEEE Transactions on Aerospaces and Electronic Systems, Vol. 28, No. 4, pp. 1105-1118, Oct. 1992

[11] Iliff, K.W.: Identification and Stochastic Control with Application to Flight Control in Turbulence,Ph.D. Dissertation, U.C.L.A., 1973 
[12] Kim, S.: A Development of Minimal Time-Change Detection Algorithm(MT-CDA) in System Parameters and Its Application to Flight Systems, Ph.D. Dissertation, U.C.L.A., 1993

[13] Lorden, G.: On the Excess over the Boundary, The Annals of Mathematical Statistics, Vol. 41, No. 2, pp. 520-527, 1970

[14] Lorden, G.: Procedures for Reacting to a Change in Distribution, The Annals of Mathematical Statistics, Vol. 42, No. 6, pp. 1897-1908, 1971

[15] Maine, R.E. and Iliff, K.W.: Identification of Dynamic Systems: Theory and Formulation, NASA Reference Publication 1138, 1985

[16] Maine, R.E. and Iliff, K.W.: Application of Parameter Estimation to Aircraft Stability and Control: The Output-Error Approach, NASA Reference Publication 1168, 1986

[17] Nelson, R.C.: Flight Stability and Automatic Control, McGraw-Hill Book Co., 1989

[18] Page, E.S.: Continuous Inspection Schemes, Biometrica, Vol. 41, pp. 100-115, 1954

[19] Page, E.S.: A test for a Change in a Parameter Occurring at an Unknown Point, Biometrica, Vol. 42, pp. 523-527, 1955

[20] Sastry, S. and Bodson, M.: Adaptive Control: Stability, Convergence, and Robustness, Prentice Hall, New Jersey, 1989

[21] Shiryayev, A.N.: Optimal Stopping Rules, Springer-Verlag, NY, 1978

[22] Siegmund D.: Sequential Analysis: Tests and Confidence Intervals, N.Y. SpringerVerlag, 1985

[23] Tartakovsky A.G.: Sequential Methods in the Theory of Information Systems, Radio i Svyaz', Moscow, 1991

[24] Tartakovsky A.G.: Asymptotically Minimax Multialternative Sequential Rule for Detection of a Disorder, Statistics and Control of Stochastic Processes, Moscow, TVP Science Publishers, 1993.

[25] Wald, A.: Sequential Analysis, Wiley, New York,1947

[26] Wald, A.: Statistical Decision Functions, Chelsea Publishing Co., 1971

[27] Wilsky, A.S.: A Survey of Design Methods for Failure Detection in Dynamic Systems, Automatica, Vol. 12, pp. 601-661, 1976 